\title{
O ICNOGÊNERO ARTHROPHYCUS DE DEPÓSITOS SUBLITORÂNEOS DA FORMAÇÃO NHAMUNDÁ (SILURIANO INFERIOR) DA BACIA DO AMAZONAS, REGIÃO DE PRESIDENTE FIGUEIREDO
}

\author{
AFONSO CÉSAR RODRIGUES NOGUEIRA*,WERNER TRUCKENBRODT ${ }^{* *}$ E EMIILIO ALBERTO AMARAL SOARES*;***
}

\begin{abstract}
THE ICHNOGENUS ARTHROPHYCUS IN SUBLITTORAL DEPOSITS OF NHAMUNDÁ FORMATION (LOWER SILURIAN), AMAZON BASIN PRESIDENTE FIGUEIREDO REGION The trace fóssil Arthrophycus alleghaniensis is reported from siliciclastic rocks of the Nhamundá Formation (Lower Silurian, Amazon Basin) near the town of Presidente Figueiredo, north of Manaus. Fácies analysis of this formation and data from literature about $A$. alleghaniensis suggest sublittoral conditions, particularly a periglacial shoreface environment, for this ichnofossil in the Amazon Basin.
\end{abstract}

Keywords: Arthrophycus, Ichnofossil, Shoreface, Nhamundá Formation, Lower Silurian, Amazon Basin

RESUMO O icnofóssil Arthrophycus alleghaniensis foi registrado em rochas siliciclásticas da Formação Nhamundá (Siluriano inferior da Bacia do Amazonas) nos arredores da cidade de Presidente Figueiredo, ao norte de Manaus. A análise faciológica desta unidade e dados bibliográficos disponíveis sobre A. alleghaniensis sugerem condições sublitorâneas, particularmente o ambiente de shoreface periglacial, para este icnofóssil na Bacia do Amazonas.

Palavras-chave: Arthrophycus, Icnofóssil, Shoreface, Formação Nhamundá, Siluriano inferior, Bacia do Amazonas

INTRODUÇÃ̃o As primeiras citações sobre o icnogênero Arthrophycus (HARLAN) HALL 1852 na Bacia do Amazonas partiram de Derby (1879) que o encontrou no sopé do Outeiro do Cachorro, margem direita do rio do Cachorro, afluente do Rio Trombetas (PA) Este autor considerou o icnofóssil como restos de plantas marinhas (Fucoides ou algas), comparando-o com a icnoespécie norte-americana Arthrophycus harlani CONRAD, de idade siluriana. Katzer (1903), entretanto, interpretou-o como impressões fósseis; enquanto Maury (1929), revisando a fauna siluriana do Rio Trombetas a partir das descrições de Clarke (1899) e com base em novas coleções, corroborou as observações de Derby (1879). Albuquerque (1922) identificou este icnogênero na Cachoeira do Moreno, localizada no rio Uatumã (AM). Décadas mais tarde, Silva (1951) registrou Arthrophycus no flanco sul da Bacia do Amazonas, no rio Tapajós (PA), enquanto Lange (1967) e Cunha \& Tarapanoff (1985) reconheceram-no, respectivamente, em estratos arenosos do poço 1-AM-1-AZ, localizado perto da confluência dos rios Negro e Solimões (AM), e em afloramentos no Município de Mazagão (AP). Todos estes registros do icnogênero Arthrophycus na Bacia do Amazonas estão restritos aos depósitos do Grupo Trombetas, de idade ordoviciana final a devoniana inicial, que reúne as formações siliciclásticas Autás-Mirim (base), Nhamundá, Pitinga e Manacapuru (topo)(Cunha et al. 1994).

Este trabalho descreve pela primeira vez o icnogênero Arthrophycus nos arredores da cidade de Presidente Figueiredo, norte de Manaus, onde ocorre em arenitos da Formação Nhamundá (Siluriano inferior), expostos principalmente em cachoeiras dos afluentes do rio Urubu (Fig. 1). A análise de fácies destes depósitos e dados bibliográficos disponíveis sobre esse icnofóssil permitiram esboçar seu habitat sublitorâneo, sob a influência de clima glacial, na Bacia do Amazonas.

CONTEXTO GEOLÓGICO-ESTRATIGRÁF1CO Os Sedimentos Nhamundá, anteriormente considerados como membro da Formação Trombetas (Lange 1967), depois que Breitbach (1957) tinha proposto informalmente este termo, foram elevados por Caputo (1984) à categoria de formação e inseridos no Grupo Trombetas. Este grupo representa a primeira sequência transgressiva-regressiva na Bacia do Amazonas e está truncada pela discordância decorrente da Orogenia Caledoniana. Mas segundo Castro et al. (1988), é possível que nas partes centrais da bacia a sedimentação marinha tenha persistido e evoluído para a sequência Urupadi e Curuá (Devoniano inferior a Carbonífero inferior).

A Formação Nhamundá é a unidade mais expressiva na região de Presidente Figueiredo (Fig. 1). Constituída por quartzo-arenitos, folhelhos e diamictitos, suas melhores exposições encontram-se em numerosas cachoeiras de rios e igarapés e chegam até $10 \mathrm{~m}$ de espessura. Depósitos mais jovens têm uma distribuição mais restrita e pertencem às formações Pitinga e Manacapuru do Grupo Trombetas e à Formação Alter do Chão (Cretáceo superior a Terciário). Ao norte da cidade de Presidente Figueiredo, a Formação Nhamundá é sobreposta bruscamente pelos folhelhos marinhos da Formação Pitinga enquanto que depósitos fluviais da Formação Alter do Chão recobrem-na discordantemente em uma faixa contínua W-E ao sul de Presidente Figueiredo e em restos isolados ao norte e leste desta cidade (Fig. 1). O contato brusco entre as formações Nhamundá e Pitinga, interpretado como superfície de inundação marinha, é um importante marco estratigráfico no Grupo Trombetas (Fig. 2). Não foi observada a interdigitação entre ambas as formações, sugerida por Grahn \& Paris (1992) em função da similaridade entre as assembleias de quitinozoários do topo da Formação Nhamundá e da base da Formação Pitinga. Folhelhos muito semelhantes aos da Formação Pitinga afloram na margem esquerda do rio Urubu, na altura do km 99 da rodovia BR-174 (Fig. 1). Análises bioestratigráfícas desta ocorrência permitiram identificar uma assembleia de quitinozoários que indica o andar Pridoliano do Siluriano correspondente à base da Formação Manacapuru (Coelho 1994). Esta ocorrência está provavelmente em contato tectônico com a Formação Nhamundá. A Formação Autás-Mirim, a unidade mais antiga do Grupo Trombetas, não aflora na região de estudo.

As primeiras informações mais precisas sobre a idade do Grupo Trombetas basearam-se na identificação do graptólito Climacograptus innonatus NICHOLSON var. brasiliensis RUEDEMANN, indicador da idade eossiluriana (Llandoveriano inferior; Maury 1929, Lange 1967). Antes desta datação, Derby (1879) já tinha considerado os fósseis do Rio Trombetas como pertencentes ao Siluriano inferior, quando comparou os exemplares de Arthrophycus com a forma $A$. harlani CONRAD do Andar Medina (Llandoveriano inferior) da América do Norte. Já Clarke (1899) estendeu a idade desta fauna até o Llandoveriano superior-Wenlockiano, enquanto que Schuchert (1906), com base nos trabalhos de Derby (1879), Ulrich (1893) e Clarke (1 899) posicionou-a no Ordoviciano superior em função da sua semelhança com a fauna do Richmond superior ou Cincinnatiano da América do Norte. Análises bioestratigráfícas mais recentes (Grahn \& Melo 1990, Quadros et al. 1990, Grahn 1991, 1992, Grahn \& Paris 1992) definiram as idades Ordoviciano superior a Lochkpviano inferior para o Grupo Trombetas e Llandoveriano a Wenlockiano inferior para a Formação Nhamundá.

LITOFÁCIES DA FORMAÇÃO NHAMUNDÁ Três principais litofácies da Formação Nhamundá foram reconhecidas na região de Presidente Figueiredo (Fig. 3) e as características são descritas resumidamente a seguir.

Foreshore Os depósitos áe foreshore têm ocorrência restrita, sendo encontradas as melhores exposições na localidade de Portal das Cachoeiras, km 15 da rodovia AM-240. São quartzo-arenitos médios a grossos, bem selecionados e arredondados, que exibem estratificação plano-paralela com truncamentos de baixo ângulo (Figs. 3 c 4A); estratos cruzados ocorrem subordinadamente. Localmente, os depósitos de foreshore são pouco bioturbados (bioturbação $<10 \%$ ) por Skolithos isp. (Figs. 3 e 4D).

A estratificação plano-paralela é atribuída à ação de fluxo e refluxo na zona de espraiamento de onda (wave swash) enquanto o trun-

* Departamento de Geociências, Universidade do Amazonas, Av. Gal. Rodrigo O. J. Ramos, 3000, Campus Universitário, CEP 69.077-000, Manaus - AM, Fax: 644-1510, e-mail: afonso@fua.br / acrnogue@usp.br

** Centro de Geociências, Universidade Federal do Pará, Campus Universitário do Guamá, CEP 66.075 -900, Belém - Pará.

*** Curso de Pós-Graduação em Geologia Geoquímica / Universidade Federal do Pará, Campus Universitário do Guamá, CEP 66.075-900, Belém - Pará. 


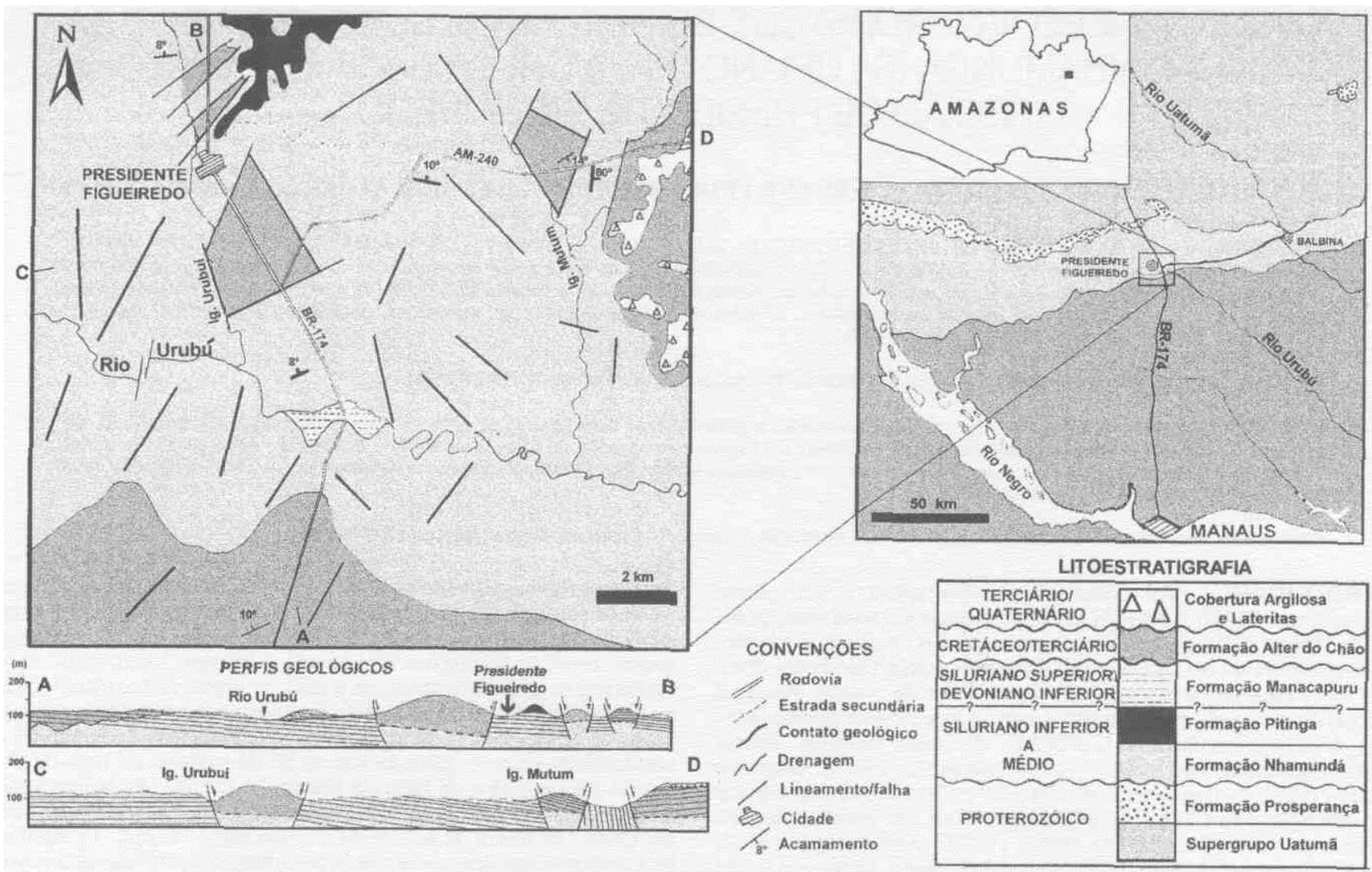

Figura l- Mapas de localização e geológico da região de Presidente Figueiredo (norte de Manaus) (Modificado de Schobbenhaus et al. J984 e Nogueira et al. 1997a).

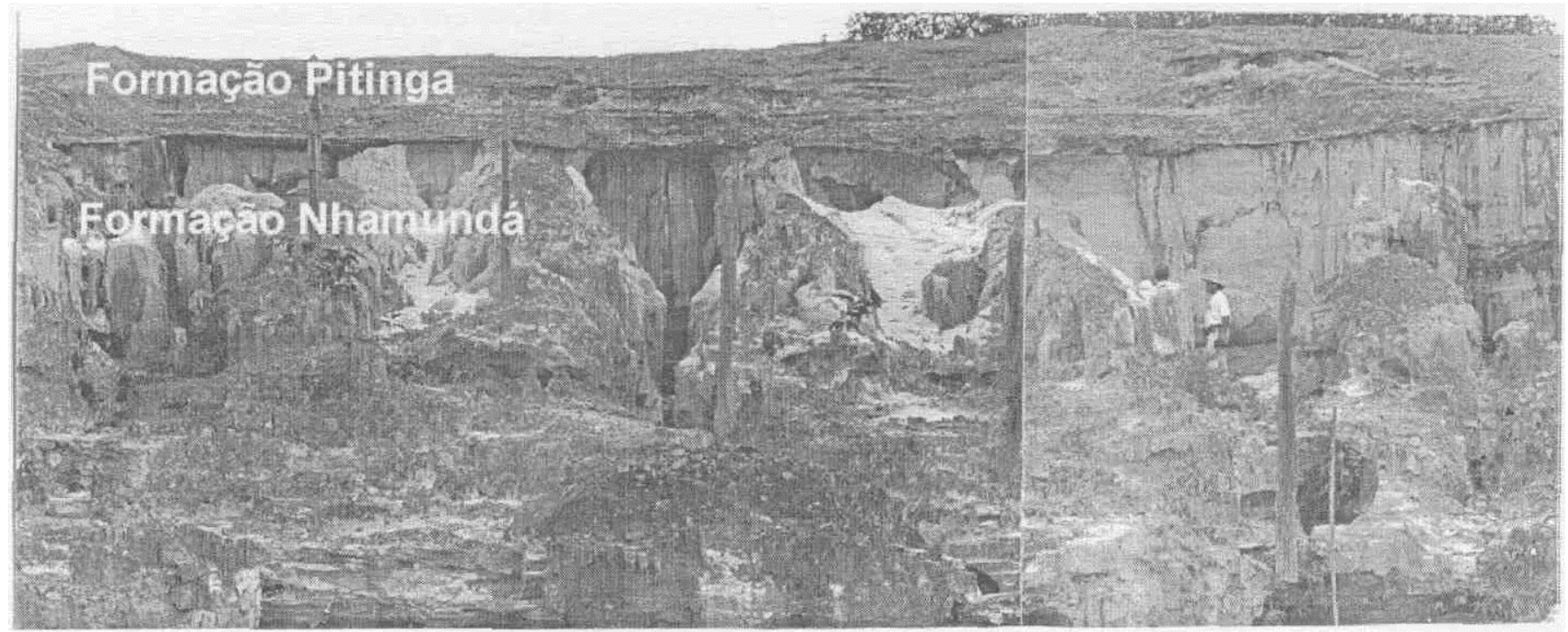

Figura 2- Superficie de inundação marinha marcando o contato entre as formações Nhamundá (arenitos) e Pitinga (folhetos) Km 108 da rodovia BR-174.

camento de baixo ângulo é interpretado como ajuste da praia a mudanças no regime de ondas ou no fornecimento de sedimento (ver Galloway \& Hobday 1996).

Shoreface Petrograficamente, os depósitos de shoreface são muito semelhantes aos de foreshore. Os quartzo-arenitos finos a grossos, com grânulos dispersos, apresentam estratificações cruzada tabular (Figs. 3 e $4 \mathrm{E})$, plano-paralela, maciça e, localmente, estratificação cruzada hummocky (Figs. 3, 4B e 4C). Pouco frequentes são pelitos, cujos estratos não ultrapassam alguns centímetros de espessura. Com exceção da estratificação cruzada hummocky, as demais estruturas caracterizam a parte superior de shoreface (Fig. 3). A parte inferior, no entanto, é pouco aflorante. Dados de paleocorrente obtidos da estratificação cruzada tabular (lâminas de foresets mergulhando para NE) são consistentes com a orientação NE-SW da paleocosta da bacia e sugerem para a origem da estratificação a migração de barras por correntes litorâneas. $\mathrm{O}$ acamamento maciço é devido à forte bioturbação $(>60 \%)$ que em grande parte é atribuída à ação de Skolithos, $\mathrm{O}$ icnogênero Arthrophycus, que ocorre na interface arenito/folhelho, é tratado em detalhe mais adiante.

Depósitos de shoreface inferior são indicados pela presença de arenitos finos com estratificação cruzada hummocky. Esta estrutura, gerada por fluxo oscilatório/combinado (Cheel \& Leckie 1993), 


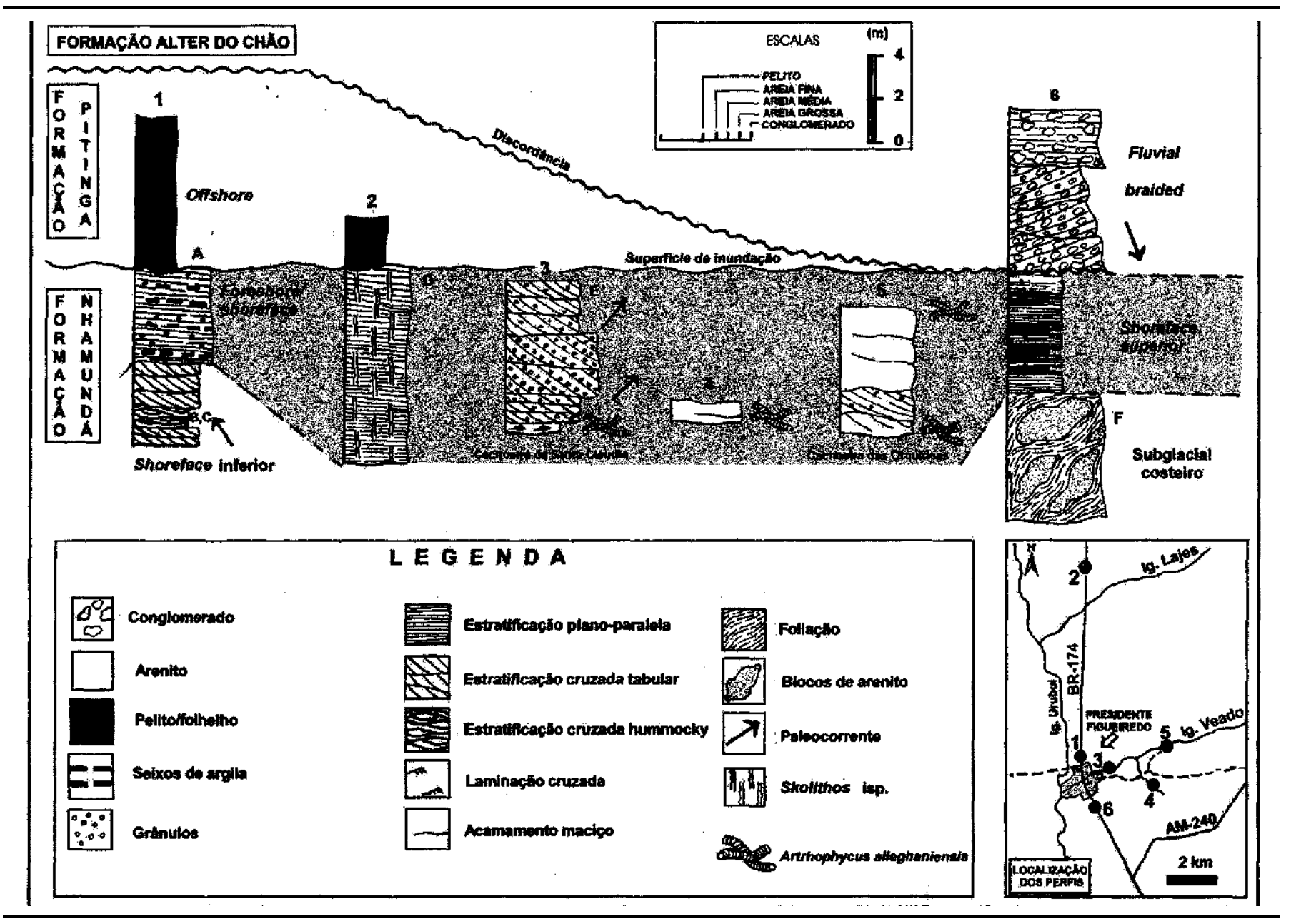

Figura 3 - Correlação liioestratigráfica e ambientes sedimentares da Formação Nhamundá na região de Presidente Figueiredo. As letras A até F, indicadas nos perfis 1 a 6 , correspondem, respectivamente, às figuras 2, 4B a 4E e 5. O mapa à direita localiza os perfis l a 6.

mostra a passagem de tempestades durante a deposição da Formação Nhamundá.

Fácies subglacial costeira $\mathrm{O}$ ambiente subglacial é evidenciado pela presença de estruturas glaciotectônicas, que incluem planos de cavalgamento de baixo ângulo e foliação subhorizontal desenvolvidos em diamictitos de constituição pelítico-arenosa (Nogueira et al. 1997b). Estas estruturas desenham um padrão anastomosado infletindo-se com mergulhos altos quando contornam porções ou blocos de quartzo-arenito fino, os quais chegam a ter comprimento métrico (Fig. 5). Boudinagem e dobras recumbentes ocorrem localmente.

As estruturas sub-horizontais, compatíveis com um campo de tensões tangenciais, sua natureza intraformacional e o fato de serem desenvolvidas em diamictitos sugerem um ambiente subglacial (Berthelsen 1979) com a geleira avançando sobre o litoral. Esta posição litorânea da fácies glacial é compatível com as demais fácies deposicionais (foreshore e shoreface) já que estas se sobrepõem em contato direto aos diamictitos.

Eventos glaciais na Formação Nhamundá foram recentemente datados por Grahn \& Paris (1992) como early middle Llandovery, early late Llandovery and latest Llandovery to earliest Wenlock através de quitinozoários em diamictitos, previamente identificados como tilitos por Carozzi et al. (1973) em testemunhos de sondagem.

ICNOGÊNERO ARTHROPHYCUS Arthrophycus alleghaniensis (HARLAN) HALL.1852 Descrição e material estudado Os exemplares deste icnofóssil representam tubos em hiporelevo positivo (hipicnia), concordantes a subconcordantes aos planos de acamamento e preenchidos com quartzo-arenito fino. Os tubos são simples e podem mostrar ramificações laterais (Fig. 6A). Já ramificações terminais, múltiplas, de até 4 tubos, são raras (Fig. 6B). Os icnofósseis alcançam até $1 \mathrm{~m}$ de comprimento e cruzam-se em ângulos ré to ou agudo. Em seção transversal, os tubos principais são elípticos a subquadráticos, com diâmetro variando de 2 a $6 \mathrm{~cm}$. Apresentam sulcos anelares transversais, espaçados regularmente ( 3 sulcos por $\mathrm{cm}$ ) que, localmente, mostram-se desgastados (Figs. 6B e C). Em alguns casos, os tubos são bilobados com sulco longitudinal mediano incipiente, denunciado por sulcos anelares parcialmente deslocados. Associadas com Arthrophycus ocorrem esporadicamente pequenas protuberâncias amendoadas (hipicnia convexa), com comprimento de até $1,5 \mathrm{~cm}$ e largura de no máximo $0,3 \mathrm{~cm}$ (Fig. 6C), similares ao icnogênero Pelecypodichnus Seilacher, 1953.

As melhores ocorrências de Arthrophycus alleghaniensis na Formação Nhamundá estão localizadas a leste da cidade de Presidente Figueiredo, na região entre as cachoeiras da Santa Cláudia (perfil 3 da Figura 3) e das Orquídeas (perfil 5 da Figura 3), incluindo um afluente esquerdo do Igarapé Veado (mapa da Figura 3). Nessas localidades foram coletadas 8 amostras de quartzo-arenito fino a médio que foram analisadas e incluídas na coleção de rochas sedimentares do Laboratório de Sedimentologia da Universidade do Amazonas sob as siglas CD-73, CD-74, CD-75, CD-76, CD-142, CD-145, CD-148 e CD-162. As amostras estão silicificadas ou mostram-se friáveis e geralmente são esbranquiçadas e vermelho-castanhas devido à pigmentação por óxidos/hidróxidos de ferro.

Interpretação paleoambientai Ao contrário dos tubos verticais de Skolithos, o icnofóssil Arthrophycus é encontrado na sola de camadas de arenito e marcando a interface arenito/pelito. Enquadrado na icnofácies Cruziana (Tabela 1), Arthrophycus é considerado monoicnoespecífico, produzido por artrópodes ou vermes (Häntzschel 1975) que escavavam túneis sub-horizontais revolvendo sedimentos à procura de alimento (feeding burrows\}? . O potencial de conservação destas estruturas, em forma de hiporelevo, se deve sobretudo a sua geração em sedimentos argilosos e posterior preenchimento por areia. A relação icnofóssil/pelito indica períodos calmos de deposição em um ambiente que era caracterizado principalmente por níveis altos de energia de corrente ou onda denunciados por arenitos com estratifi- 

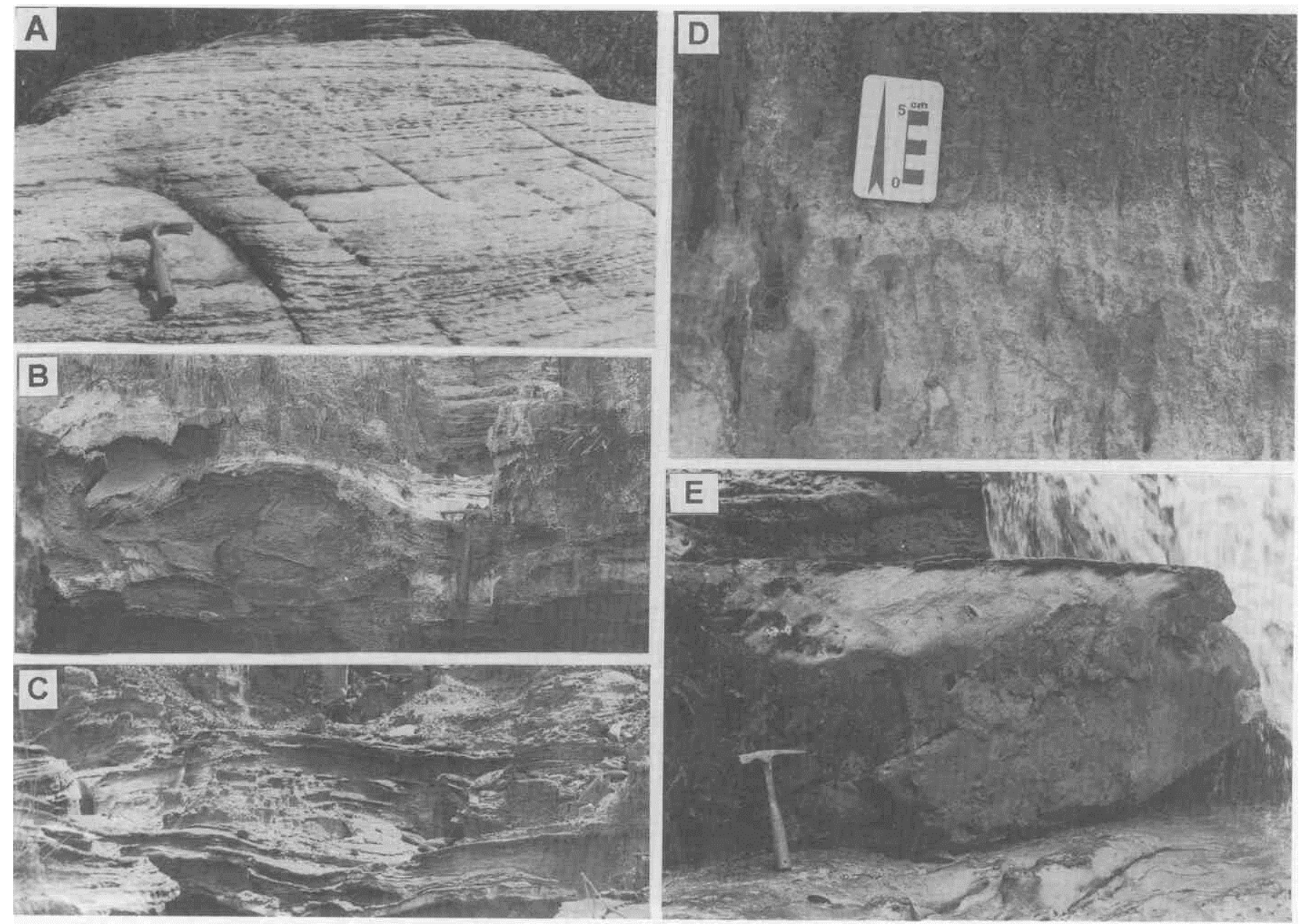

Figura 4 - Estruturas sedimentares da Formação Nhamundá. A) Estratificação plano-paralela com truncamentos de baixo ângulo. B e C) Estratificação cruzada hwnmocky (detalhe do perfill da figura 3). D) Skolitlios isp. (detalhe do perfil 2 da figura 3). E) Estratificação cruzada tabular (detalhe do perfil 3 da figura 3).

cações cruzada e plano-paralela e marcas onduladas. A associação de Arthrophycus a níveis descontínuos de arenito fino a médio com marcas onduladas intercalados a arenitos com estratificação cruzada sotopostos à fácies de foreshore, indica um ambiente de shoreface (sublitoral), provavelmente superior, para este icnofóssil. Esta interpretação é de acordo com a maioria dos dados bibliográficos disponíveis (Tabela 1), mas que mostram também a ocorrência de Arthrophycus em baixios de offshore (Selley 1970, 1985), depósitos de crevasse splay em baia interdistributária (Eagar 1985) e em ambiente fluvial braided distai (Cotter 1983). Mesmo que um ambiente não marinho possa ser posto em dúvida, já que segundo Pickerill et al. (1991) Arthrophycus é sempre registrado em sucessões marinhas e demarca a linha de costa no Paleozóico, sendo relacionado a condições litorâneas rasas (Boucot 1975), os organismos que produziram este icnofóssil suportavam a influência de água doce em ambientes litorâneos (Legg 1985, Eagar 1985). Condições de salinidade reduzida são igualmente sugeridas pelo menos para a parte de shoreface da Formação Nhamundá, com base na ausência de corpos fósseis e da presença de Pelecypodichnus isp. que segundo Hakes (1985) e Pienkowski (1985) é conhecido em sedimentos de água salobre. O recuo do gelo eossiluriano da área de estudo poderia ter contribuído no fornecimento de grandes volumes de água doce.

A posição do polo Sul na região meridional do Brasil durante o Eossiluriano (Caputo 1984, Grahn \& Caputo 1992) implica em condições periglaciais para o ambiente sublitorâneo de Arthrophycus. Um clima frio na Bacia do Amazonas é também corroborado pela ausência de sedimentação carbonática durante todo o EoPaleozóico.

Amplitude estratigráfica Embora Arthrophycus alleghanien-sis seja conhecida na Bacia do Amazonas apenas em depósitos siluri-

anos, sua distribuição estratigráfica a nível global é mais ampla e estende-se do Cambriano ate o Carbonífero (Tabela 1). Na Bacia do Paraná, este icnofóssil além de ocorrer na Formação Vila Maria do Siluriano inferior (Burjack \& Popp 1981, Borghi et al 1996), foi recentemente registrado também em depósitos sotopostos à Formação Vila Maria, atribuídos ao Ordoviciano (Fernandes \& Borghi 1996).

CONCLUSÕES O icnofóssil Arthrophycus alleghaniensis na Formação Nhamundá (Siluriano inferior), região de Presidente Figueiredo, ocorre como hiporelevo positivo na base de camadas de arenito e marcando a interface arenito/pelito. A análise faciológica destes depósitos indica o ambiente de shoreface, provavelmente, shoreface superior, para este traço-fóssil. Além disso, a ausência de corpos fósseis e a presença de Pelecypodichnus, conhecido em sedimentos de água salobre, sugerem para Arthrophycus um ambiente de shoreface com salinidade reduzida. Indicativos climáticos periglaciais para o habitat deste icnofóssil são fornecidos pela associação dos depósitos de shoreface com os diamictitos subglaciais caracterizados por planos subhorizontais de cavalgamento e foliação.

Embora Arthrophycus seja registrado na Bacia do Amazonas apenas em depósitos silurianos, sua distribuição estratigráfica a nível mundial é mais ampla e estende-se do Cambriano até o Carbonífero. $\mathrm{Na}$ Bacia do Paraná, Arthrophycus ocorre tanto em sedimentos silurianos como ordovicianos.

Agradecimentos À Universidade do Amazonas pelo apoio financeiro e ao estudante Jackson Douglas Paz pelo acompanhamento no campo, bem como a Leonardo Borghi, Mário Vicente Caputo e aos dois relatores anónimos da Revista Brasileira de Geociências pelas criticas e sugestões ao texto original. 

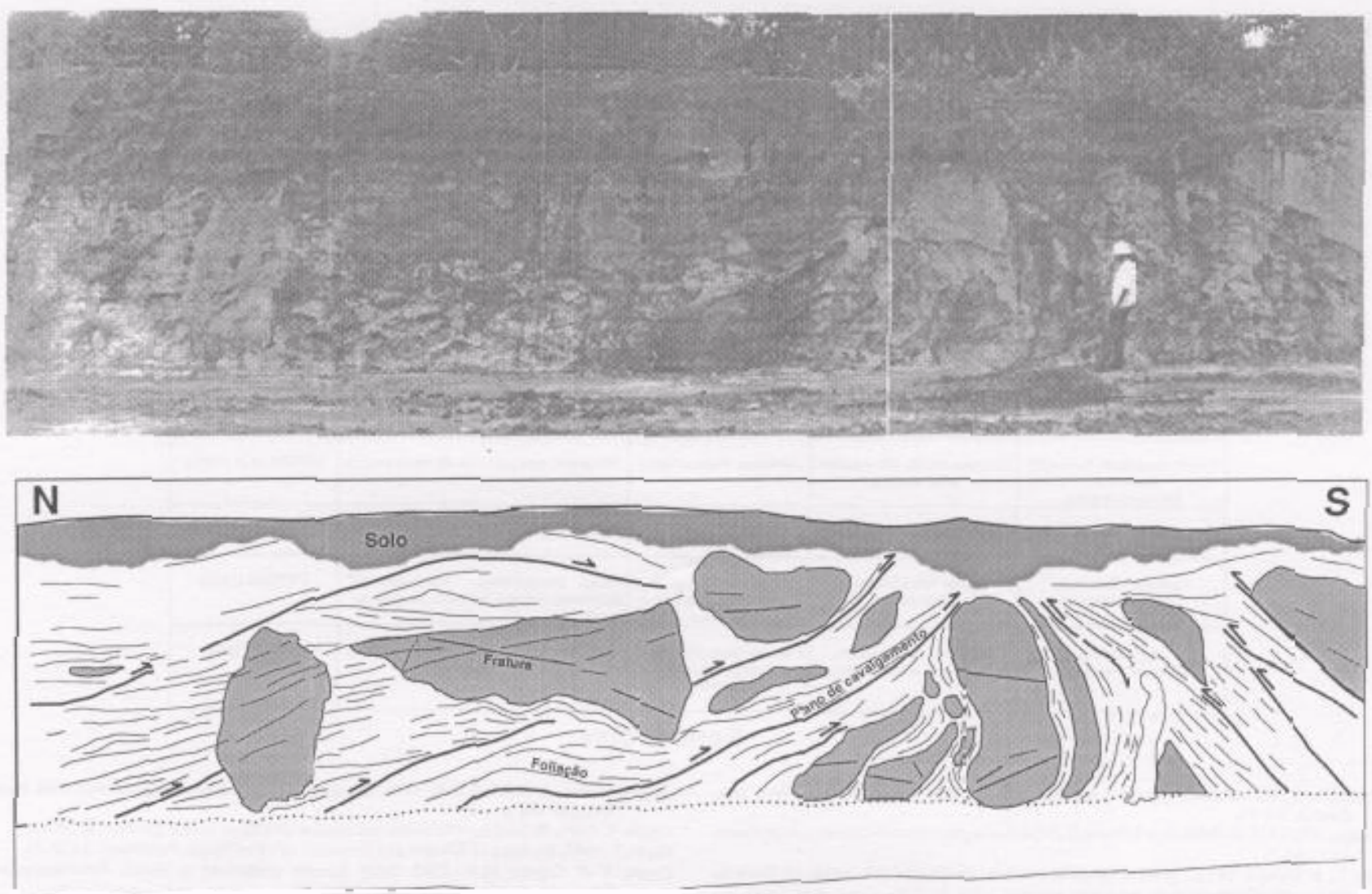

Figura 5 - Estruturas glaciotectônicas na Formação Nhamundá. Foliação e planos de cavalgamento, desenvolvidos em diamictito, envolvendo blocos de quartzo-arenito s fino (detalhe do perfil 6 da figura 3).

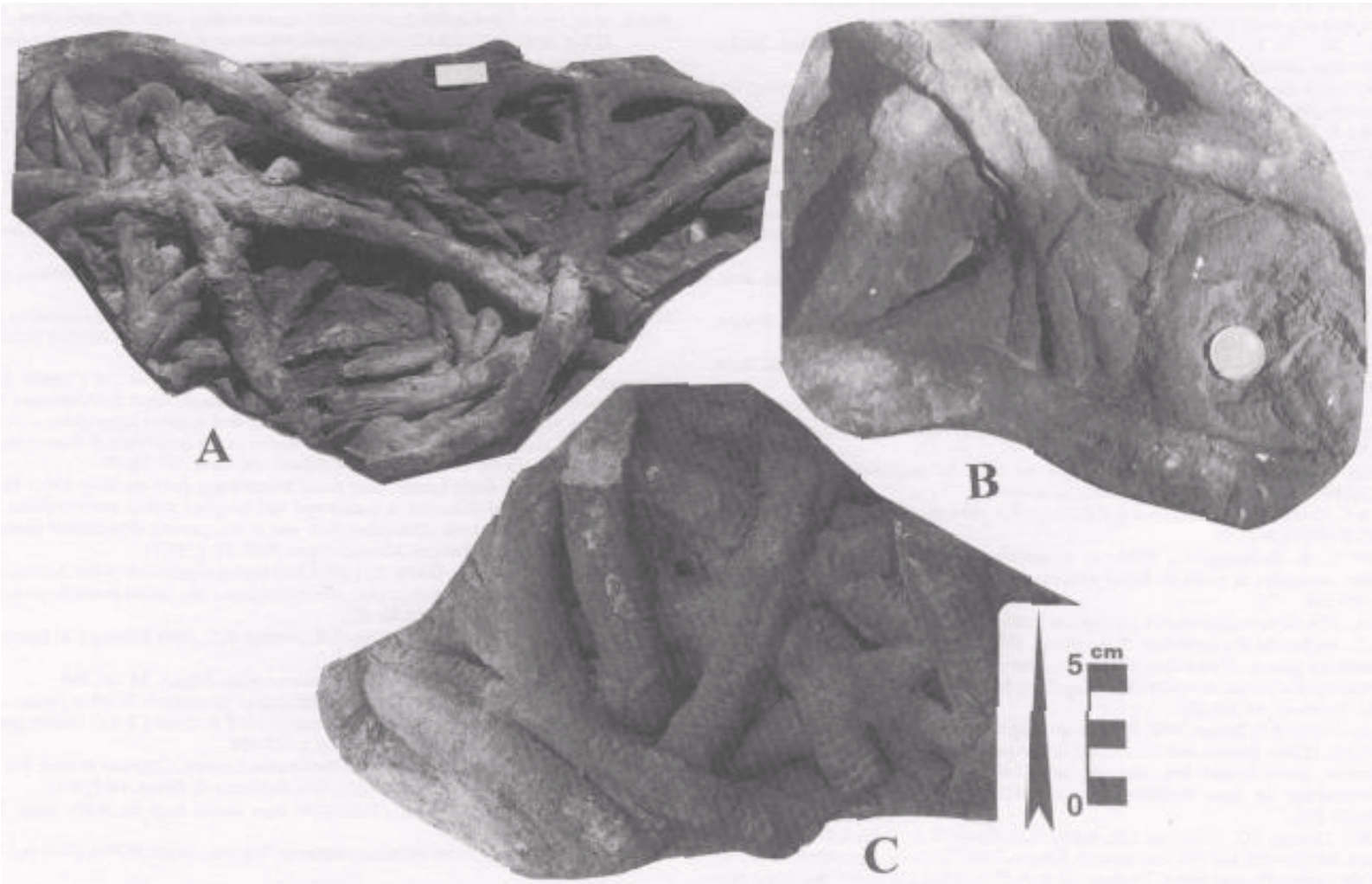

Figura 6 - Arthrophycus alleghaniensis. A) Aspecto geral mostrando tubos ramificados em hiporelevo. B) Ramificação múltipla do traço. C) Estruturas anulares transversais e, localmente, sulco mediano incipiente no traço; as pequenas protuberâncias hipicniais amendoadas (setas) são atribuidas a Pelecypodichnus isp. 
Tabela l - Interpretações estratigráfica e paleoambiental para Arthrophycus.

\begin{tabular}{|c|c|c|c|c|}
\hline $\begin{array}{l}\text { UNIONDE } \\
\text { ESTRATIOALFICA }\end{array}$ & LITOTPO (S) & ICNOCENOSE & PALEOAMEIENTE & AUTORES \\
\hline $\begin{array}{l}\text { Areniloe ef folthelhos de Ovillo } \\
\text { (Cambriano Médio). }\end{array}$ & Arentto siftico & Cruzlane, Fhyeodes & Delta com influencla de maré & LEGG (1985) \\
\hline $\begin{array}{l}\text { Sucessåa paleozálea da } \\
\text { Bacia do Kuira } \\
\text { (Eambriano-Dewoniano) }\end{array}$ & $\begin{array}{l}\text { Sithtoss o arentitos finos, } \\
\text { oventuainnente arentos } \\
\text { médtos. }\end{array}$ & Cruziana. & $\begin{array}{l}\text { Platatorna, submare e canais da } \\
\text { submare, transiçao fluwial braided a } \\
\text { submare. }\end{array}$ & $\begin{array}{c}\text { TURNER \& BENTON } \\
\text { (19B3) }\end{array}$ \\
\hline $\begin{array}{c}\text { Formaçă La Tinta } \\
\text { (Ordovikieng) }\end{array}$ & Sullto e arenito & Cruzlana & Sublitoral & ACENHOLAZA (1978) \\
\hline $\begin{array}{c}\text { Formaçăo Um Sahm } \\
\text { (Ordoviciano) }\end{array}$ & Arendilos e follhethos & Cruziana, Skolfthos & Babcios de olishore, fitoraneo e della & SELEY (1970, 1985) \\
\hline $\begin{array}{l}\text { Formaçăo Vila Maria } \\
\text { (Siluriano Inlertor) }\end{array}$ & Sithito arenito & $\begin{array}{l}\text { Skofithos, PPlanollfes, } \\
\text { PChondtifes }\end{array}$ & $\begin{array}{l}\text { Sublitoral relativamente raso: glacio. } \\
\text { marknto sob aça do lompestados }\end{array}$ & $\begin{array}{l}\text { BUPUACK \& POPP } \\
\text { (1991):BOAGH: } \\
\text { MOREARA(19OG) }\end{array}$ \\
\hline $\begin{array}{c}\text { Clinch Sandstone, Formaçá } \\
\text { Rockwood, } \\
\text { (Sifuriano Inferion) }\end{array}$ & $\begin{array}{l}\text { Intertace arenilo fino pentlos } \\
\text { (mud drapes). }\end{array}$ & Pkanolloses, Palaopphycus & Shoreface, com pertodos do baixa energla & DRIESE of al (1991) \\
\hline $\begin{array}{c}\text { Grupo Medina } \\
\text { (Siluriano Interion) }\end{array}$ & Arenilos e pellios intercalados & Daedatiss, Planothos & Baixtios arenosos do submarí a Intermare & DUKE of a'(1991) \\
\hline $\begin{array}{l}\text { Formaçắo Tuscarora } \\
\text { (Silluriano Inlerior) }\end{array}$ & $\begin{array}{l}\text { Arenito. fino e lothethos } \\
\text { (interface) }\end{array}$ & $\begin{array}{c}\text { Monocraterion; } \\
\text { Ausophycus, Chondirles, } \\
\text { Skath hos }\end{array}$ & $\begin{array}{l}\text { Filuvial braided clistal, sanctwaves de } \\
\text { platalormia marinha, estuabrio, planicle de } \\
\text { mare? }\end{array}$ & COTTER (1983) \\
\hline $\begin{array}{l}\text { Marsdeniano } \\
\text { (Carbonffero Madilo) }\end{array}$ & $\begin{array}{l}\text { Arenito quartzoso interta- } \\
\text { minado com siltitios e pelitos }\end{array}$ & $\begin{array}{c}\text { Palaeophycus, Planolltes, } \\
\text { Palocypodichnus, } \\
\text { Phycedes }\end{array}$ & Crevasse splay em bala interdistributária & EAGAR (1985) \\
\hline
\end{tabular}

\section{Referências}

Albuquerque, R. 1922. Reconhecimentos geológicos no Vale do Amazonas: Serv. Geol. Min, Boletim, 3:1-84

Aceffolaza, F.G. 1978. El Paleozóico Inferior da Argentina scgun sus trazas fosilcs. Ameghiniana, 15:15-64.

Borghi, L. \& Moreira, M.I.C. 1996. A ocorrência inédita de sistema ordoriciano na Bacia do Paraná, em afloramentos de seu bordo noroeste. 1996. In: SBG, Cong. Brás. Gcol., 39, Salvador, Anais, 1:145:147

Borghi, L.; Moreira, I.C.; Fernandes, A.C.S. 1996. A ocorrência do icnogcncro Arthrophycus Hall, $1852 \mathrm{~cm}$ Chapada dos Guimarães, Estado de Mato Grosso. An. Acad. Brás. Ci, 68:274-275.

Berthelsen, A . 1979. Recumbent folds and boudinagc structures fortncd by subglacial shcar: an example of gravity tectonics. Geol. Mijnbouw, 58:253-260.

Breilbach, J. W. 1957. Geology of Rio Cajari, Paleozoic outcrop belt, lowcr Amazon. Belém, Petrobras. (Relatório Inédito 210-A).

Boucot, A.J. 1975. Evolution and extinction rate contrais: Developments in palaeontology and stratigraphy. Amsterdam, Elsevier, 427 p.

Burjack, M.I.A. \& Popp, M.T.B. 1981. A ocorrência do icnogêneroArthrophycus no Paleozóico da Bacia do Paraná. Pesquisas, 14:163-168.

Caputo, M.V. 1984. Stratigraphy, tectonics, paleoclimatology andpaleogeography of nortttem basins ofBrazil. Univcrsity of Califórnia, Santa Barbara, PhD Thesis, 583 p.

Carozi, A.V.; Pamplona, H.R.P.;

Castro, J.C.; Contreiras, C.J.A. 1973. Ambientes deposicionais e evolução tectono-sedimentar da seção elástica paleozóica da Bacia do Médio Amazonas. In: SBG, Cong. Brás. Geol., 27, São Paulo, Anais, 3:279-314. Castro, J.C.; Eiras, J.F.;

Caputo, M.V. 1988. Paleozóico do Rio Tapajós. In: SBG, Cong. Brás. Geol., 35, Belém, Anais.p. 27-44. Roteiro das Excursões.

Cheel, R.J. \& Leckic, D.A. 1993. Hummocky cross-stratification. Sedimentology Review, 1:103-122.

Clarkc, J.M. 1899. A fauna siluriana superior do rio Trombetas, estado do Pará, Brasil. Arch. Mus.Nac., 10:1-48

Cotter, E. 1983. Shelf, paralic, and fluvial environmenls and eustatic sea-level fluctuations in the origin of the Tuscarora Formation (Lower Silurian) of Central Pcflnsylvania. J. Sed. Petrol., 53:25-49.

Coelho, G.L. 1994. Relatório final de estágio na área de palinologia. PETROBRAS -CENPES/DIVEX/SEBIPE, 22p. Relatório interno.

Cunha, P.R.C.; Gonzaga, F.G.; Coutinho, L.F.C; Fcijó, F.J. 1994. Bacia de Amazonas. B. Geoc PETROBRAS, 8:47-55. Cunha, B.C.C. \& Tarapanoff, I. 1985. A mesopotâmia

Preto Cajari (AP) e a seção

siluro-devoniana na Bacia do Baixo Amazonas. In:SBG, Simp. Geol. Amaz., 2, Anais, 1:106-120.

Derby, O.A. 1879. Contribuicões para a geologia do baixo Amazonas. Areh Mus. Afac.2:77-104.

Driese, S.G.; Fischer, M.W.' Easthouse, K.A.; Marks, G.T; Gogola A.R.'Schoncr, A.E. 1991. Model for génesis of shorcfacc and shclf sandstone sequences, soulhern Appalachians: palaeoenvironmental reconstruction of an Early Silurian shelf system. Spec. Púbis. Int. Ass. Sediment., 14:309-338.

Duke, W.L; Fawcctt, P.J.; Brusse, W.C. 1991. Prograding shoreline deposits in the Lower Silurian Medina Group, Ontario and New York: storm-and tide- influenced scdimcntation in a shallow cpicontinental sca, and the origin of cnigmatic shorc-normal channcls encapsulated by open shallow-marine deposits. Spec. Púbis. Int. Ass. Sediment., 14:339-375.

Eagar, R.M.C; Baines, J.G.; Collinson, J.D.; Hardy, P.G.; Okolo, S.A.; Pollard, J.E. 1985. Trace fóssil assemblagcs and their occurcnce in Silcsian (Mid-Carboniferous) dcltaic scdiments of the Central Penninc Basin, England. In: H.A. Curran (cd.) Biogenic structures: their use in interpreting depositionalenvironments. Tulsa, Soe. Econ. Paleont. Mineral., Spec. Publ.35,p.99-149.

Fernandes, A.C.S. \& Borghi, L. 1996. Comentários sobre o icnogênero Arthrophycus Hall 1852 e seu registro nas bacias sedimentares brasileiras. In: SBG, Simp. Sul Americano do Siluro-Devoniano, Ponta Grossa, 1:131-139.
Galloway, W.E. \& Hobday, D.K. 1996. Terrigenous clastic depositional systems. 2 ed. Berlin, Springcr, $489 \mathrm{p}$

Grahn, Y. 1991. Ordovician chitinozoa and biostratigraphy ofBrazil. Geobios, 6: 703-723.

Grahn.Y. 1992. Revision of Silurian and Dcvonian strata ofBrazil. Palinology, 16:35-61. Grahn, Y \& Caputo, M.V. 1992. Early Silurian glaciations in Brazil. Palaeogeography, Palaeoclimatology, Palaeoecology, 99:9-15.

Grahn, Y \& Melo, J.H.G. 1990. Bioestratigrafia dos quitinozoários do Grupo Trombetas nas Faixas Marginais da Bacia do Amazonas (área dos rios Trombetas c Xingu/ Relatório Interno da PETROBRAS, $11 \mathrm{p}$

Grahn, Y. \& Paris, F. 1992. Age and correlalion of the Trombetas Group, Amazonas Basin, Brazil. Rev. Micropaleontologie, 35:197-209.

Hakcs, W.G. 1985. Trace fossils from brackish-marine shales, Upper Pennsylvanian of Kansas, U.S.A. In: H.A. CURRAN (ed.) Biogenic structures: their use in interpreting depositional environments. Tulsa, Soe. Econ. Paleont. Mineral., Spec. Publ. 35, p.21-35.

Häntzschcl, W. 1975. Trace fóssil and problemática. In: C. TEICHERT (ed.) Treatise on invertebrate palaeontology. Boulder, Geol. Soe. Am., (Part W, Miscellanea), 269p.

Katzer, F. 1903. Grundzüge der Geologie des unteren Amazonasgebietes., Leipzig, Verlag Max Wcg, 296-p.

Langc, F.W. 1967. Subdivisão bioestratigráfica e revisão da coluna siluro-devoniana da Bacia do Baixo Amazonas. Atas do simpósio sobre a Biota Amazônica (Geociências), 1:215-326.

Legg, I.C. 1985. Trace fossils from a Middlc Cambrian deltaic sequence, north Spain. In: H.A. CURRAN (cd.) Biogenic structures: their use in interpreting depositional environments. Tulsa, Soe. Econ. Paleont. Mineral., Spec. Publ. 35, p. 151-165.

Maury, C.J. 1929. Uma zona de graptólitos do Llandovery Inferior no Rio Trombetas, Estado do Pará, Brasil. Serv. Geol. Min.: Monogr., .:7:1-53.

Nogueira, A.C.R.; Souza, V.; Soares, E.A.A. 1997a. Contribuicão à tectônica cenozóica da região de Presidente Figueiredo, norte de Manaus. In: SBG, Simp. Nac. Estudos Tectônicos, 6 , Pirinópolis,. Boi. resumos expandidos, p.123-125.

Nogueira, A.C.R.; Soares, E.A.A.; Souza, V.; Trukenbrodt, W; Caputo, M.V.1997b. Estruturas glaciotcctõnicas na Formação Nhamundá, Siluriano da Bacia do Amazonas. In: SBG, Simp. Nac. Estudos Tectônicos, 6, Pirinópolis,. Boi. resumos expandidos, p. 153-155.

Pickcrill, R.K.; Fillon, D.; Brcnchley, P.J. 1991. A note on the occurrence of Arthrophycus in the Bell Island Group of castern Ncwfoundland. Atl. Geol., 27: 73-77.

Pienkowski, G. 1985. Early Liassic trace fóssil assemblages from the Holy Cross Mountains, Poland: Their distribution in continental and marginal marinc environments. In: H.A. Curran (ed.) Biogenic structures: their use in interpreting depositional environments. Tulsa, Soe. Econ. Paleont. Mineral., Spec. Publ. 35, p. 37-51.

Quadros, L.P.; Melo, J.H.G.; Grahn, Y. 1990. Carta bioestratigráfica da Bacia do Amazonas. In G. Beurlcn, \& L.P. Quadros (cds.) Bioestratigrafia das bacias paleozóicas brasileiras Rio de Janeiro, PETROBRAS. Scho

Schobbcnhaus, C; Campos, D.A.; Dcrzc, G.R.; Asmus, H.E. 1984. Geologia do Brasil. DNPM, Brasilia, $501 \mathrm{p}$.

Schuchert, C. 1906. Geology of the lowcr Amazon rcgion. JGeoL, 14:722-746.

Selley, R.C. 1970. Ichnology of Paleozoic sandstones in the southern desert of Jordan: a study of trace fossils in their sedimentologic context. In: T.P. Crimes \& J.C. Harper (eds.) Trace fossils. Liverpool, Geological Society, p.477-488.

Selley, R.C. 1985. Ancient sedimentary environments. London, Chapman \& Hall, 317 p.

Silva, S.O. 1951. Siluriano no Rio Tapajós. Rev. da Escola de Minas, 16(5):9-11.

Turner, B.R. \& Bcnton, M.J. 1983. Palaeozoic trace fossils from the Kufra Basin, Lybia. J. Paleont., 57:447-460.

Ulrich, A. 1893. Palaeozoischc Versteinerungen aus Bolivien. Neues Jb.Min. Geol. Pai. Beil. Bd., 8:5-116.

Manuscrito A- 987

Recebido em 4 de junho de 1998 Revisão dos autores em 15 de fevereiro de 1999 Revisã o aceita em 20 de fevereiro de 1999 